\title{
Pyramidal Cells in Piriform Cortex Receive Convergent Input from Distinct Olfactory Bulb Glomeruli
}

\author{
Alfonso Apicella, ${ }^{1,2}$ Qi Yuan, ${ }^{1,2}$ Massimo Scanziani, ${ }^{1,2,3,4}$ and Jeffry S. Isaacson ${ }^{1,2}$ \\ ${ }^{1}$ Center for Neural Circuits and Behavior, ${ }^{2}$ Neuroscience Department, School of Medicine, ${ }^{3}$ Neurobiology Section, Division of Biology, and ${ }^{4}$ Howard Hughes \\ Medical Institute, University of California, San Diego, La Jolla, California 92093-0634
}

Pyramidal cells in piriform cortex integrate sensory information from multiple olfactory bulb mitral and tufted (M/T) cells. However, whether M/T cells belonging to different olfactory bulb glomeruli converge onto individual cortical cells is unclear. Here we use calcium imaging in an olfactory bulb-cortex slice preparation to provide direct evidence that neurons in piriform cortex receive convergent synaptic input from different glomeruli. We show that the combined activity of distinct glomerular pathways recruits ensembles of pyramidal cells that are not activated by the individual pathways alone. This cooperative recruitment of cortical neurons only occurs over a narrow time window and is a feature intrinsic to the olfactory cortex that can be explained by the integration of converging, subthreshold synaptic input. Cooperative recruitment enhances the differences between cortical representations of partially overlapping input patterns and may contribute to the initial steps of olfactory discrimination.

\section{Introduction}

In rodents, signals from 1000 different types of odorant receptors (ORs) map onto 1800 glomeruli in the olfactory bulb and olfactory sensory neurons (OSNs) expressing one unique OR project onto 50-100 mitral and tufted (M/T) cells within each glomerulus (Mombaerts et al., 1996). Individual odorants are detected by different, but partially overlapping, combinations of ORs, leading to the activation of multiple glomeruli in the olfactory bulb (Wilson and Mainen, 2006). Ultimately, information from combinations of activated M/T cells belonging to different glomeruli is proposed to undergo a synthetic process in cortical brain regions to generate odor perception. However, the stage in cortical processing in which information from different glomeruli is initially combined is not clear. The anterior piriform cortex (APC) is a three-layered cortical region that receives direct input from $\mathrm{M} / \mathrm{T}$ cell axons within the lateral olfactory tract (LOT) (Neville and Haberly, 2004) and thus a potentially important site for the integration of information from combinations of glomerular pathways.

Previous studies revealed the basic features governing the integration of LOT synaptic input and spike output of layer $2 / 3$ (L2/3) principal cells in APC slices. Individual L2/3 cells receive inputs from multiple M/T cells (Franks and Isaacson, 2006; Bathellier et al., 2009) and though some single LOT fibers make strong connections, the majority are sufficiently weak such that the coincident activity of multiple inputs must be integrated for

\footnotetext{
Received May 29, 2010; revised Sept. 7, 2010; accepted Sept. 8, 2010.

This work was supported by the National Institute on Deafness and Other Communication Disorders (DC04682, A.A., Q.Y., and J.S.I.) and the National Institute of Mental Health (MH70058, M.S.).

Correspondence should be addressed to Jeffry S. Isaacson, Center for Neural Circuits and Behavior, Neuroscience Department, University of California, San Diego, Room 213, 9500 Gilman Drive, La Jolla, CA 92093-0634. E-mail: jisaacson@ucsd.edu.

DOI:10.1523/JNEUROSCI.2747-10.2010

Copyright $\odot 2010$ the authors $\quad 0270-6474 / 10 / 3014255-06 \$ 15.00 / 0$
}

L2/3 principal cells to reach spike threshold (Franks and Isaacson, 2006; Bathellier et al., 2009). Thus, the firing of principal cells in APC reflects the integration of subthreshold excitatory synaptic responses generated by multiple afferent fibers. This integration could lead to the nonlinear recruitment of ensembles of active cortical neurons, since combined activity of distinct afferent pathways should produce firing in subpopulations of neurons that only generated subthreshold synaptic responses to individual afferent pathways alone.

The integrative properties of cortical neurons could play a major role in odor coding. However, a fundamental unanswered question in olfaction is whether individual L2/3 principal cells in piriform cortex receive converging input from $\mathrm{M} / \mathrm{T}$ cells belonging to different glomeruli. Despite the lack of evidence directly addressing this question, odor representations in APC are often proposed to reflect the integration of inputs from multiple glomeruli by single neurons. Indeed, this scenario is thought to account for observations that individual piriform cortex neurons in vivo respond to different odorants (Wilson, 2003; Rennaker et al., 2007; Poo and Isaacson, 2009) and that different odorants activate unique, but partially overlapping, ensembles of L2/3 cells (Stettler and Axel, 2009). The convergence of inputs from multiple glomeruli onto cortical neurons is also thought to underlie findings that subpopulations of neurons are only responsive to odorant mixtures, but not to the individual components (Zou and Buck, 2006; Stettler and Axel, 2009). However, these features of odor coding could result from processing steps at a number of levels in the olfactory system. For example, OSNs also respond to multiple odorants, different odorants activate unique but partially overlapping ensembles of OSNs, and odorant mixtures evoke OSN responses that can differ from the responses to individual components (Wilson and Mainen, 2006). Further downstream, processing within intrabulbar circuits (Wilson, 2008) or recurrent connections between cortical pyramidal cells (Neville 
and Haberly, 2004) could also underlie in vivo observations attributed to the convergence of different glomeruli onto single cortical neurons. Thus, the degree to which cortical odor representations reflect the convergence and integration of distinct glomerular pathways is unclear.

In this study, we use a brain slice preparation containing the olfactory bulb and cortex to explore features of cortical ensemble coding. We image action potential (AP)-dependent calcium transients in APC pyramidal cells and test directly the coding strategies underlying activation of cortical ensembles by unique combinations of olfactory bulb inputs. We provide evidence that individual APC principal cells receive converging synaptic input from different glomeruli. We also show that mixtures of mitral cell inputs recruit larger ensembles of pyramidal neurons whose composition strongly differs from those recruited by the individual components. This nonlinear behavior of olfactory cortex increases differences between cell ensembles representing activity from combinations of overlapping sensory inputs.

\section{Materials and Methods}

Experiments followed approved national and institutional guidelines for animal use. Postnatal day 9 (P9)-P14 mice (ICR) were anesthetized with ketamine $(100 \mathrm{mg} / \mathrm{kg})$ and xylazine $(10 \mathrm{mg} / \mathrm{kg})$ and decapitated. The cortices and attached olfactory bulbs were placed into ice-cold artificial CSF (aCSF) containing the following (in $\mathrm{mm}$ ): $83 \mathrm{NaCl}, 2.5 \mathrm{KCl}, 0.5$ $\mathrm{CaCl}_{2}, 3.3 \mathrm{MgSO}_{4}, 1 \mathrm{NaH}_{2} \mathrm{PO}_{4}, 26.2 \mathrm{NaHCO}_{3}, 22$ glucose, and 72 sucrose equilibrated with $95 \% \mathrm{O}_{2}$ and $5 \% \mathrm{CO}_{2}$. For bulb-cortex slices, horizontal sections $(500-600 \mu \mathrm{m})$ were cut using a vibrating slicer (Vibratome) and incubated at $34^{\circ} \mathrm{C}$ for $30 \mathrm{~min}$. For isolated cortex slices, the bulbs were removed and parasagittal slices were cut $(400 \mu \mathrm{m})$. Slices were superfused with aCSF containing the following (in $\mathrm{mM}$ ): $119 \mathrm{NaCl}, 2.5$ $\mathrm{KCl}, 2.5 \mathrm{CaCl}_{2}, 1.3 \mathrm{MgSO}_{4}, 1 \mathrm{NaH}_{2} \mathrm{PO}_{4}, 26.2 \mathrm{NaHCO}_{3}, 22$ glucose equilibrated with $95 \% \mathrm{O}_{2}$ and $5 \% \mathrm{CO}_{2}$ and viewed with an upright microscope using differential interference contrast (DIC) optics. Image acquisition ( $494 \mathrm{~nm}$ excitation, $2 \times 2$ binning, $25-50 \mathrm{~Hz}$ ) was performed with a cooled-CCD camera system (T.I.L.L. Photonics). All experiments were conducted at $30-32^{\circ} \mathrm{C}$, and data are mean \pm SEM.

Bulb-cortex slices were bulk loaded with Oregon Green-1 AM (OG-1, $10-20 \mu \mathrm{M}$, with $0.02 \%$ Pluronic F-127) at $34^{\circ} \mathrm{C}$ for $30 \mathrm{~min}$. In experiments using isolated cortex slices, L2/3 was "painted" with OG-1 (50 $\mu \mathrm{M})$ by gently blowing the dye solution through a pipette ( $\sim 2 \mu \mathrm{m}$ tip) at the slice surface. Focal glomerular stimulation was evoked using monopolar electrodes placed in aCSF-filled pipettes ( $1-2 \mu \mathrm{m}$ tip diameter, $10-40 \mathrm{~V}$, $0.2 \mathrm{~ms}$ stimuli). Pipettes were positioned in two distinct glomeruli visualized by DIC optics to avoid stimulation of the overlying olfactory nerve layer. Glomeruli in the mouse olfactory bulb have an average diameter of $85 \mu \mathrm{m}$ (Royet et al., 1988), and the two stimulation sites were separated by $>250 \mu \mathrm{m}$ to prevent stimulation of overlapping glomeruli. We confirmed the focal nature of glomerular stimulation using loose patch recordings of APs in mitral cells directly below individual glomeruli (supplemental Fig. 1, available at www.jneurosci.org as supplemental material). Mitral cell activation declined sharply when the stimulation electrode was moved $\geq 50 \mu \mathrm{m}$ laterally, and when it was moved $>100$ $\mu \mathrm{m}$, mitral cells were unresponsive $(n=6)$. In imaging experiments, we delivered trains of four pulses at $50 \mathrm{~Hz}$ for glomerular stimulation to mimic odor-evoked firing of mitral cells in vivo (Cang and Isaacson, 2003), and stimulus strength was adjusted to evoke reliable but nonoverlapping calcium signals at each glomerular site. Similar results were obtained with single stimulus pulses, but fewer APC neurons were reliably recruited on individual trials (not shown). LOT stimulation was delivered via bipolar steel electrodes, and all responses were evoked at 0.1 Hz. Electrophysiological data were recorded with a Multiclamp 700A (Molecular Devices) and collected and analyzed using Axograph.

Imaging data were analyzed using Image (NIH). Activity maps of cell ensembles were constructed from 3-6 stimulus trials (supplemental Fig. 2 , available at www.jneurosci.org as supplemental material). All images from an individual experiment were processed identically. The first 5-10 image frames ( $200-400 \mathrm{~ms})$ following the stimulus were averaged to generate the peak $\mathrm{d} F / F$ signal for individual trials (without background subtraction). Individual peak $\mathrm{d} F / F$ images were then averaged together to represent the cell ensembles activated by each input pathway alone or those stimulated together. The resulting peak $\mathrm{d} F / F$ images were bandpass filtered (to reduce diffuse signals) and smoothed. Cells were detected from these processed images using a template-based detection criterion based on their size $(\sim 20 \mu \mathrm{m}$ diameter) and intensity ( $>50 \%$ of background) to yield a final binary image of active cells.

We compared the cells detected using the template-based approach with those determined from analyzing calcium transients in individual trials (supplemental Fig. 2, available at www.jneurosci.org as supplemental material). We used the binary images of detected cells in response to simultaneous stimulation of two pathways as ROIs to measure the $\mathrm{d} F / F$ responses of the same cells on individual trials ( 155 cells, $n=3$ slices, 5 trials). We used a threshold of $\mathrm{d} F / F>2 \%$ ( $1 \%$ above background) for determining successes from failures on individual trials. This was derived from the mean and $\mathrm{SD}$ of $\mathrm{d} F / F$ signals for failures $(0.5 \pm 0.7 \%)$ and single APs $(4.0 \pm 0.9 \%)$ in loose-patch recordings $(n=6)$. On average, $\sim 60 \%$ of cells in the average activity maps following simultaneous stimulation were active on $5 / 5$ stimulus trials. Only a small fraction of detected cells $(\sim 4 \%)$ were not resolved on individual trials, presumably because the activity map revealed some cells with a low signal-to-noise ratio on single trials. On average, $\sim 70 \%$ of cells detected during simultaneous stimulation were never active on individual trials ( $0 / 5$ trials) when each pathway was stimulated independently. We further tested the identification of ensembles recruited by independent stimulation by comparing the number of cells detected from the average activity maps to those that were active on $\geq 1 / 5$ stimulus trials. There was no difference in the cells detected using these two approaches (paired $t$ test, $p=0.7, n=6$ pathways from 3 experiments), confirming that the activity maps are a good reporter of active cell ensembles. We estimated the number of APs in active cells by analyzing the distribution of $\mathrm{d} F / F$ values for cells that responded on individual trials ( $n=6$ slices, $\sim 200$ cells). The distribution was well described by the sum of three equidistant peaks corresponding to one (77\%), two (19\%), or three (4\%) APs. Thus, the majority of cells responded with one AP under our conditions.

\section{Results}

To visualize ensembles of cells in piriform cortex activated in response to stimulation of sensory inputs, we imaged brain slices loaded with the calcium indicator Oregon Green-1 AM (Ikegaya et al., 2005). We first used bulbocortical slices (Balu et al., 2007), a preparation where the olfactory bulb remained functionally connected to the piriform cortex (Fig. $1 A_{1}$ ). A stimulating electrode was placed in the mitral cell layer of the olfactory bulb, and we imaged the response to brief trains of stimuli ( 4 pulses, $10 \mathrm{~ms}$ interstimulus interval) in L2/3 of anterior piriform cortex (Fig. $1 A_{2}$ ). Stimulation of mitral cells evoked a marked rise in fluorescence of L2/3 of piriform cortex (Fig. $1 A_{2}, A_{3}$ ). Furthermore, we could resolve bright spots in $\mathrm{d} F / F$ image sequences that reflected calcium signals in the cell bodies of individual L2/3 neurons (Fig. $1 A_{2}$ ). The stimulus-evoked signal in L2/3 was abolished following application of the glutamate receptor antagonists NBQX (10 $\mu \mathrm{M})$ and $\operatorname{APV}(50 \mu \mathrm{M})$ (Fig. $\left.1 A_{3}\right)$; however, imaging in layer I directly below the LOT revealed a robust stimulus-evoked response due to calcium influx into LOT presynaptic terminals (Fig. $1 A_{3}$ ). These results indicate that we can monitor long-range synaptic activation of cortical cells via LOT inputs in bulb-cortex slices, and we constructed maps of pyramidal cell activity by averaging together the peak $\mathrm{d} F / F$ images from multiple trials (Fig. $1 B)$. Loose-patch recordings confirmed that stimulus-evoked calcium transients in the cell bodies of L2/3 neurons were detected only when trials generated APs (Fig. 1C) ( $n=10$ cells). Whole-cell current-clamp recordings revealed that activated cells had regular spike firing properties ( $n=5$, not shown), and the 

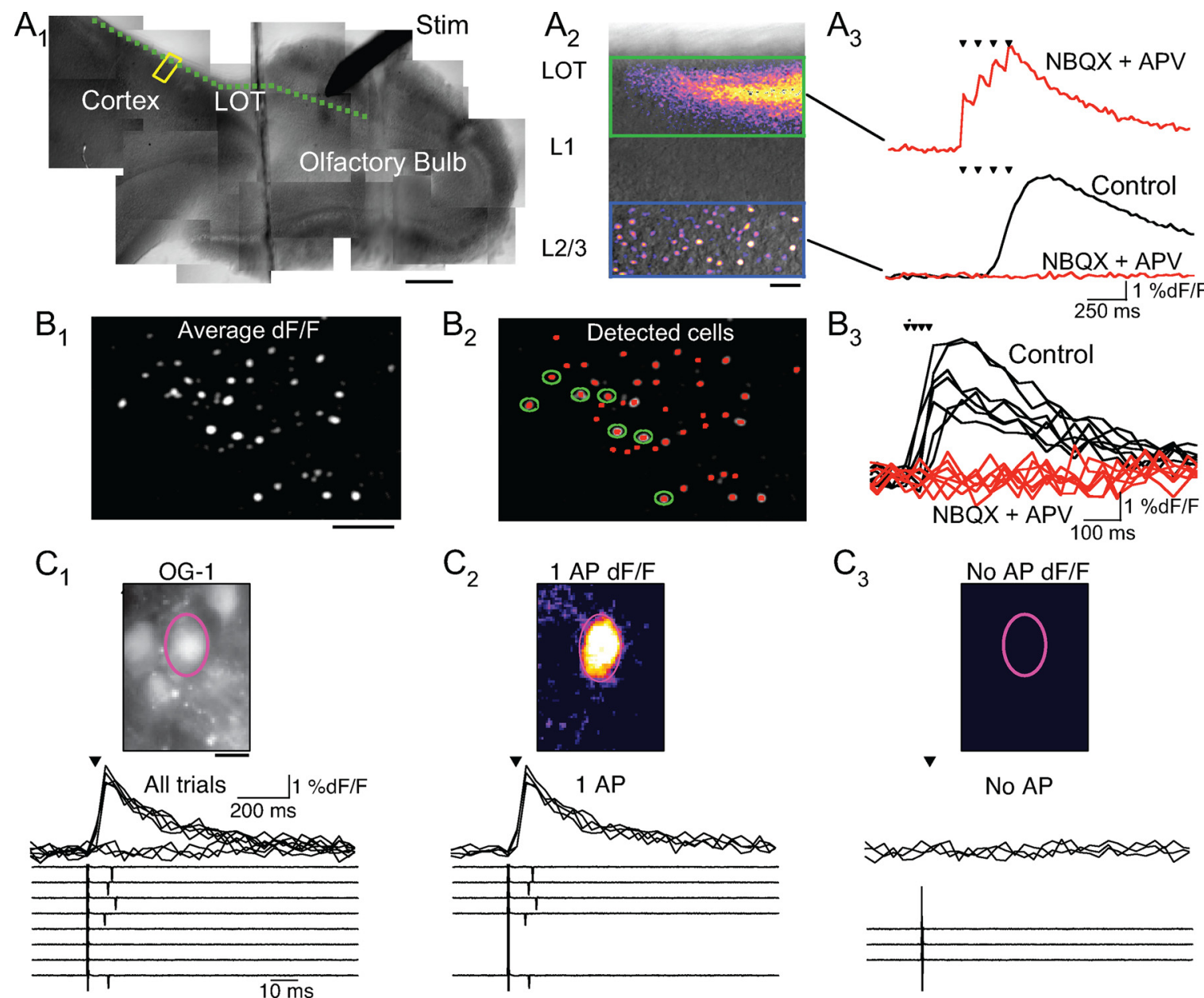

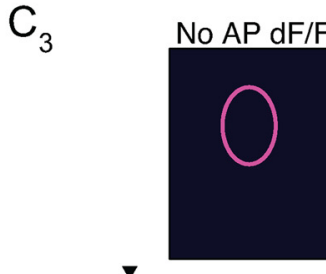

No AP

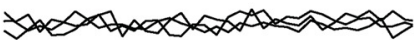

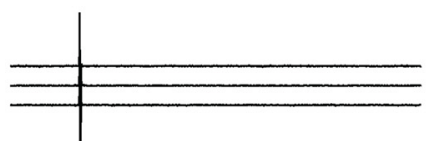

Figure 1. Visualizing cortical ensembles activated by mitral cells in olfactory bulb- cortex slices. $A_{1}$, A stimulating electrode (Stim) is in the mitral cell layer and the dashed line is the route of the LOT. Scale bar, $200 \mu \mathrm{m}$. $A_{2}$, DIC image of the region of cortex outlined in $A_{1}$. Superimposed is the peak $\mathrm{d} F / F$ response of the cell layer region (blue box) in response to a stimulus train (4 pulses, 10 $\mathrm{Hz}$ ) delivered to the mitral cell layer under control conditions and the response in layer 1 (green box) in the presence of NBQX and APV. Scale bar, $50 \mu \mathrm{m}$. $A_{3}$, Bottom traces, Field dF/F response of the cell layer region before (black) and after (red) addition of NBQX (10 $\mu \mathrm{M})$ and APV $(50 \mu \mathrm{M})$. Top trace, Response in layer 1 in the presence of glutamate antagonists. Arrowheads indicate stimulus train. $\boldsymbol{B}_{1}$, Average dF/Fimage of layer $2 / 3$ from five trials of stimulation in the olfactory bulb. Scale bar, $50 \mu \mathrm{m}$. $\boldsymbol{B}_{2}$, Activity map (red) overlaid on $\mathrm{d} F / F$ image showing detected cells. $\boldsymbol{B}_{3}$, Single-trial $\mathrm{d} / F / F$ traces of cells outlined in $B_{2}$ before (black) and after (red) application of glutamate receptor antagonists. $C_{1}$, Top, OG-1 labeled cells in L2/3. The outlined cell was targeted for loose-patch recording. Scale bar, $12.5 \mu \mathrm{m}$. Middle, LOT stimulation (arrowhead) evoked calcium transients in 5/8 trials. Bottom, Simultaneously loose patch recording revealed APs in $5 / 8$ trials. $C_{2}$, Top, Average $\mathrm{d} F / F$ from the field in $\boldsymbol{C}_{1}$ for all trials in which an AP was detected. Middle and bottom, All trials in which the cell fired an action potential coincided with a calcium transient. $\boldsymbol{C}_{3}$, Top, Average $\mathrm{d} F / F$ for trials with no APs. Middle and bottom, Trials in which the cell did not fire an AP coincided with a lack of a calcium transient.
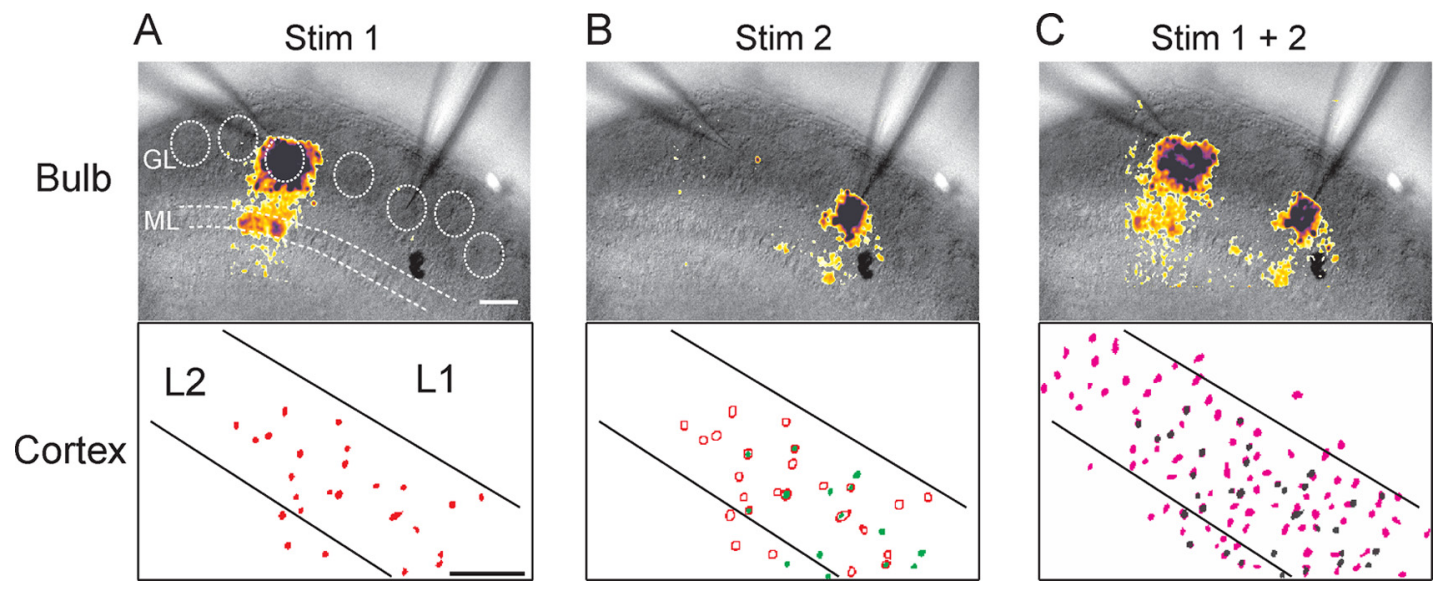

Figure 2. Cooperative recruitment of cortical neurons in response to stimulation of distinct olfactory bulb glomeruli. Sequential imaging of peak dF/Fresponses to stimulation in the olfactory bulb glomerular layer (top) and cell activity maps in piriform cortex (bottom) are shown. $\boldsymbol{A}$, Focal stimulation in one glomerular region (Stim1) activates an ensemble of cells in cortex (red). $\boldsymbol{B}$, Focal stimulation of a different glomerular region (Stim2) activates another ensemble of cells (green) some of which were also activated by Stim1 (open circles are cells from $\boldsymbol{A}$ ). $\boldsymbol{C}$, Simultaneous stimulation of the two glomerular regions (Stim1 +2 ) recruits a large ensemble of cells that were not activated by each pathway alone (black cells). Scale bar, $100 \mu \mathrm{m}$. 

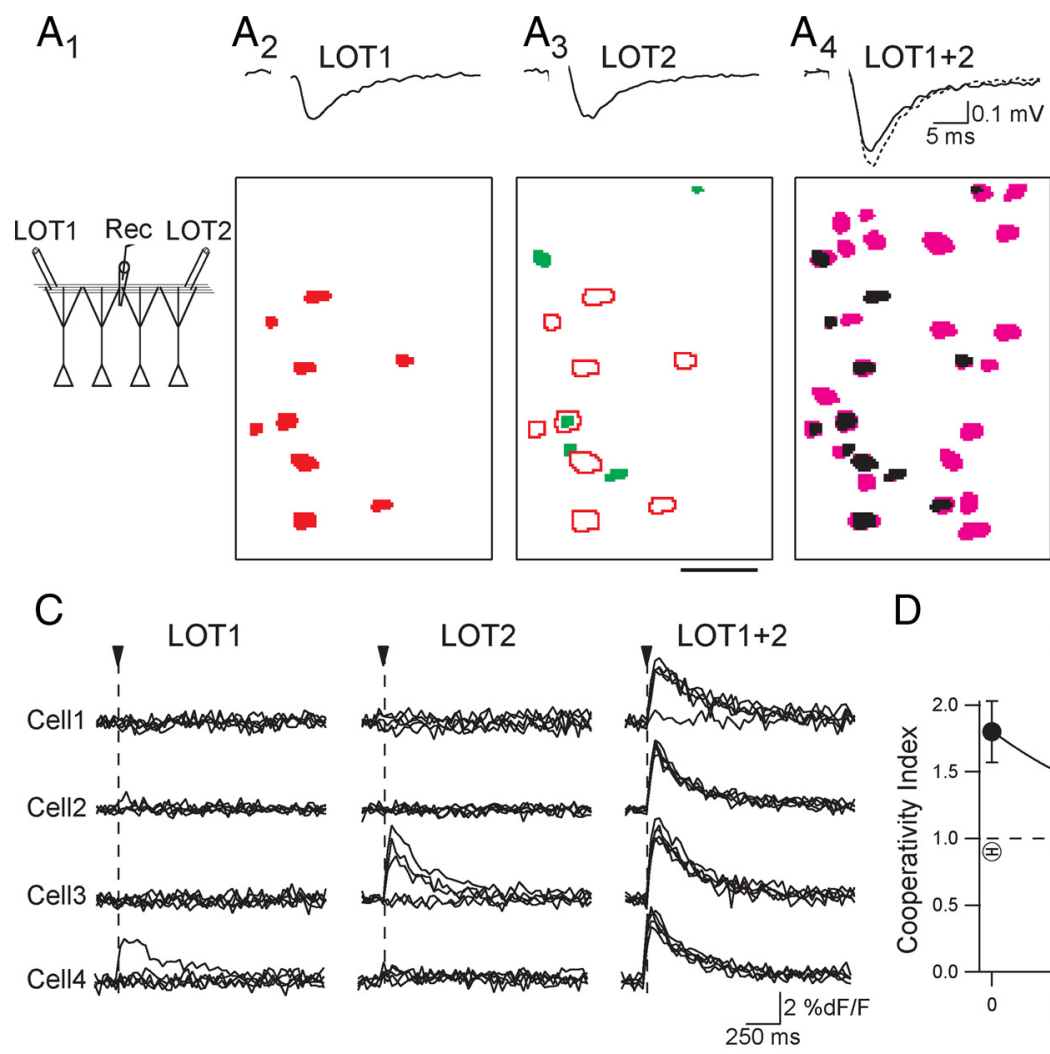

B
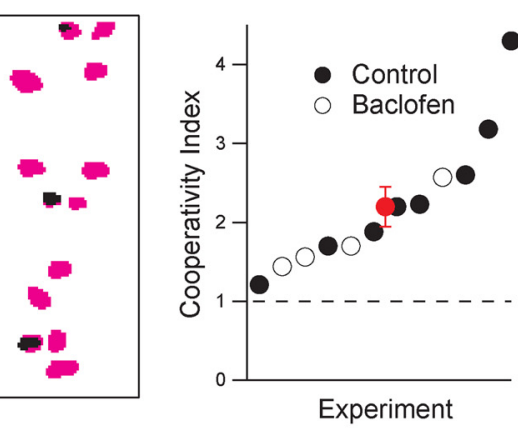

C

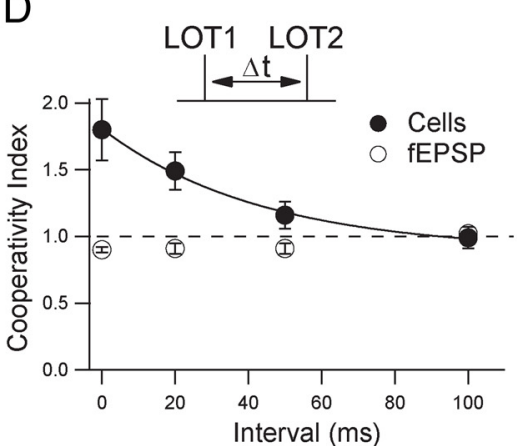

Figure 3. Cooperative recruitment of cells in isolated slices of piriform cortex. $A_{1}$, Schematic of stimulating electrodes (LOT1 and LOT2) and field recording electrode. $A_{2}$, Average EPSP (top) of the trials used to make the cortical activity map (bottom) in response to stimulation of LOT1. $A_{3}$, EPSP and activity map (green) in response to stimulation of the LOT2 pathway. Cells responding to LOT1 are superimposed (red outlines). $\boldsymbol{A}_{4}$ EPSP evoked by stimulation of LOT1 +2 superimposed with the sum of the individual EPSPs (dotted trace). Cells recruited by coincident stimulation (magenta) are superimposed with those evoked independently by the two pathways (black). Scale bar, $50 \mu \mathrm{m}$. B, Summary of the cooperative recruitment of cells under control conditions (filled circles, $n=$ 8 ) and in the presence of baclofen $(50 \mu \mathrm{m}$, open circles, $n=4)$. Average value from all experiments shown in red. C, Individual kinetic plots of $\mathrm{d} F / F$ from four cells in one experiment $(5$ consecutive trials per cell). Arrowhead indicates LOT stimulation. D, Summary of the linearity of EPSPs (open circles) and cell ensembles (filled circles) in response to different LOT stimulus intervals ( $n=6$ slices). The supralinear response of pyramidal cells is fitted with an exponential ( $\tau=40 \mathrm{~ms}$ ).

high density of principal cells relative to GABAergic interneurons in L2/3 (Neville and Haberly, 2004) makes it likely that the vast majority of imaged neurons are pyramidal cells.

Do axons of $\mathrm{M} / \mathrm{T}$ cells belonging to different glomeruli converge onto individual pyramidal cells? To address this question, we placed two focal stimulating electrodes in the olfactory bulb glomerular layer to activate distinct and non-overlapping glomerular regions (Fig. 2). Calcium imaging confirmed that stimulation of the two widely spaced sites activated unique sets of glomeruli (Fig. $2 A, B$ ). After monitoring glomerular activation in the olfactory bulb, we imaged the APC in the same bulb-cortex slices. We searched for regions (fields $\sim 200 \times 300 \mu \mathrm{m}$ ) of L2/3containing cells that were activated in response to one of the two glomerular stimulation sites (Fig. 2A). Invariably, the same region also contained cells activated by the other glomerular stimulation site (Fig. 2B). Stimulation of each glomerular pathway recruited distinct ensembles of pyramidal cells ( $12 \pm 2$ cells per ensemble, $n=6$ slices); however, a fraction of the activated pyramidal cells were common to both inputs $(10 \pm 4 \%)$ (Fig. $2 B$ ). Together, these findings show that different glomeruli can drive activity in overlapping areas of olfactory cortex and that $\mathrm{M} / \mathrm{T}$ cell inputs arising from different glomeruli converge onto common pyramidal cells.

A consequence of converging feedforward input and the temporal summation of subthreshold synaptic responses is that combined activation of distinct afferent pathways should drive AP firing in larger numbers of neurons than are recruited by the individual pathways alone. Indeed, synchronous stimulation of the two glomerular sites recruited many new pyramidal cells that were not activated when either of the two glomerular pathways were stimulated independently (Fig. 2C). We quantified the recruitment of pyramidal cells in response to combined stimuli using a cooperativity index [\# of cells activated by pathway 1 and pathway 2 together/(\# of cells activated by pathway 1 alone + pathway 2 alone - overlapping cells activated by both pathways)]. On average, the number of cells recruited by a combination of stimuli was two times larger than the sum of cells (adjusted for overlap) recruited by either pathway alone $(2.1 \pm 0.3, n=6)$. Thus, coincident activation of distinct glomerular pathways recruits novel ensembles of pyramidal cells that were not represented by the individual pathways alone.

To rule out the possibility that the additional pyramidal cells recruited by coactivation of two pathways reflected increased $\mathrm{M} / \mathrm{T}$ cell recruitment by bulbar circuits, we studied slices of piriform cortex that were not attached to the bulb. In slices of isolated APC we used stimulating electrodes in the LOT to directly activate two sets of M/T cell axons. L2/3 cells were imaged at the midpoint of the two stimulating electrodes, and a recording electrode was placed in layer I to monitor the field EPSP (fEPSP) (Fig. $3 A_{1}$ ). We used weak stimulus intensities to produce sparse activation of cortical cells similar to what we observed with focal stimulation of olfactory bulb glomeruli. We determined the independence of the two sets of LOT inputs by comparing the sum of the fEPSPs evoked by each LOT pathway alone to the fEPSP 


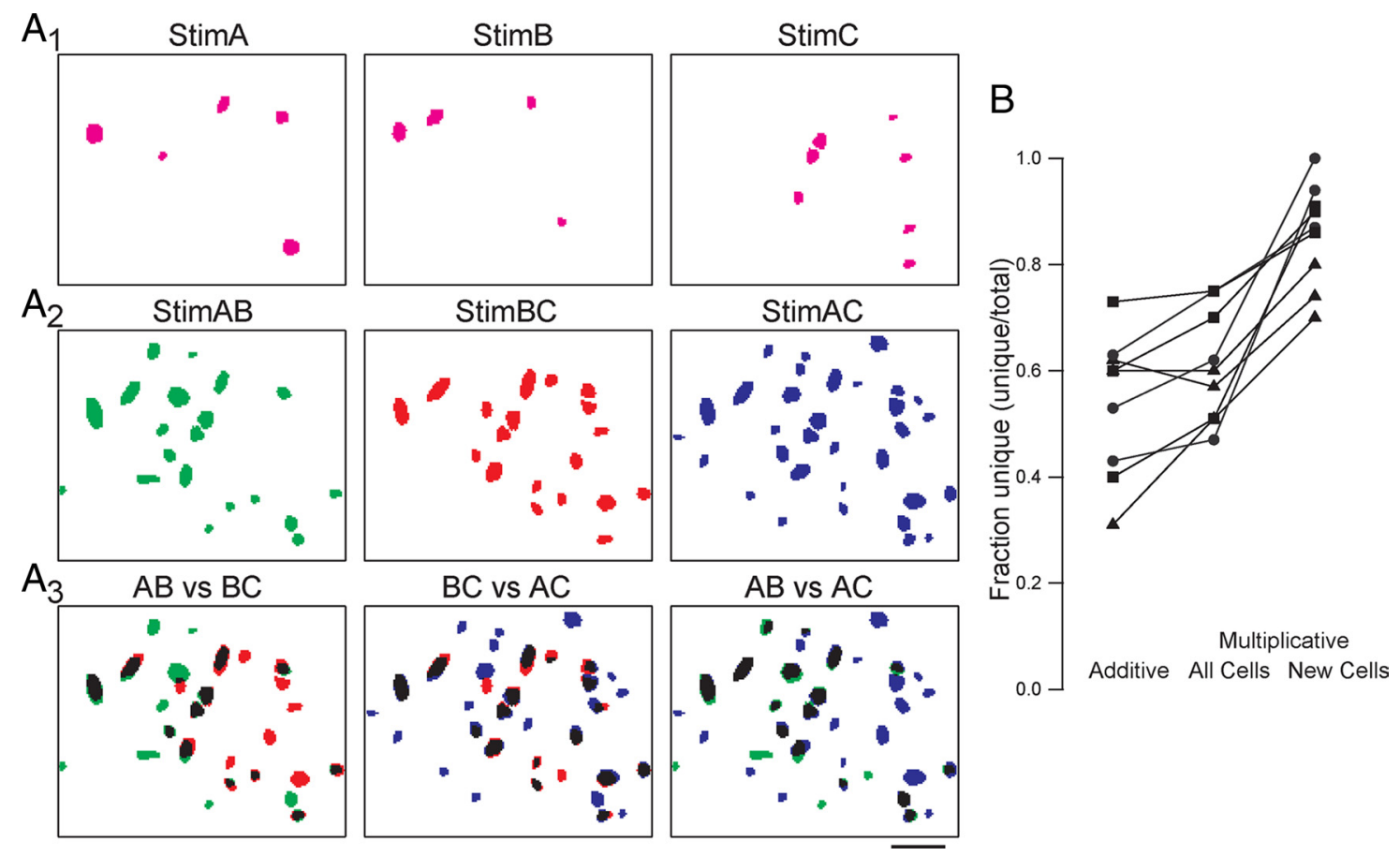

Figure 4. Cooperative recruitment of pyramidal cells enhances the difference between cell ensembles representing different input combinations. $A_{1}$, Cell ensembles activated by stimulation of three different LOT pathways. $A_{2}$, Cooperative responses to each of the three possible combinations of inputs. $A_{3}$, Overlay of all input combinations reveals cells that are unique to each combination. Cells that are common to each pairwise combination are shown in black. Scale bar, $50 \mu \mathrm{m}$. $\boldsymbol{B}$, Results from three experiments (different symbols) plotting the unique fraction of each input combination (Stim $A B, B C, A C$ ). The unique fraction of each combination is first plotted as the sum of the independent pathways (Additive). The unique fraction of the cell ensembles is greater when the pathways were stimulated simultaneously (Multiplicative, All Cells). The cells selectively recruited with simultaneous stimulation were the most unique for each input combination (Multiplicative, New (ells).

when both pathways were activated simultaneously. The degree of input overlap of the two LOT pathways determined from the fEPSPs was only $8 \pm 2 \%(n=12)$. Similar to results using stimulation of olfactory bulb glomeruli, we found that each LOT pathway activated distinct ensembles of L2/3 cells (Fig. $3 A_{2}, A_{3}$ ). Simultaneous stimulation of the two LOT pathways recruited more cells than the sum of the cells activated by each pathway alone (Fig. $\left.3 A_{4}\right)$ in all slices tested $(n=8)$ (Fig. $3 B$ ). This result was not the consequence of an increased activation of LOT fibers, as demonstrated by the independence test (see above), and rules out the involvement of bulbar circuitry in the cooperative recruitment of pyramidal cells by distinct glomerular pathways.

While pyramidal cells are directly excited by LOT inputs, associational connections between neighboring pyramidal cells (Neville and Haberly, 2004) are another potential source of cortical excitation. We considered the possibility that recurrent excitation between pyramidal cells could underlie the cooperative recruitment of cell ensembles. To address this issue, we performed experiments in the presence of the $\mathrm{GABA}_{\mathrm{B}}$ receptor agonist baclofen $(50 \mu \mathrm{M})$, which causes a potent presynaptic inhibition of associational inputs but has no effect on direct LOTevoked responses (Franks and Isaacson, 2005). However, baclofen did not prevent the activation of additional cells by the simultaneous stimulation of independent pathways ( $n=4$ slices) (Fig. $3 B$ ), suggesting that recurrent excitation is not required for cooperative recruitment. On average, simultaneous stimulation of two LOT pathways yielded a cooperativity index of $2.21 \pm 0.25$ $(n=12)$. In addition to recruiting more cells, combined stimulation also increased the reliability of cells that responded only intermittently to each pathway alone (Fig. $3 C$ ). Together, these experiments indicate that the supralinear recruitment of cells by coactivation of independent sensory pathways does not require cooperative interactions in the bulb; rather, it results from the integration of convergent LOT inputs onto pyramidal cells within APC.

Over what temporal interval do LOT pathways cooperate in the recruitment of pyramidal cells? We stimulated two LOT pathways either simultaneously or with progressively increasing temporal separation (Fig. 3D). The recruitment of new cells was maximal during simultaneous stimulation $(\Delta t=0 \mathrm{~ms})$ of the two pathways and lessened as the interval between the two stimuli increased. On average, cooperative recruitment of new cells decayed with a time constant of $40 \mathrm{~ms}$ ( $n=6$ slices). These results indicate that novel pyramidal cell ensembles arising from combinations of olfactory inputs occur only within a narrow time window.

How unique are the ensembles of additional pyramidal cells recruited by the simultaneous activity of two pathways to that particular combination of inputs? To address this question, we used three stimulating electrodes ( $\mathrm{A}, \mathrm{B}$, and $\mathrm{C}$ ) placed in the LOT, which independently recruited three different ensembles of pyramidal cells (Fig. $4 A_{1}$ ), and visualized the ensembles recruited by the three input combinations $\mathrm{AB}, \mathrm{AC}$, and $\mathrm{BC}$ (Fig. $4 A_{2}, A_{3}$ ). Note that each input combination shares half of the input with any other combination. The three representations were matched against each other and the fraction of unique neurons determined for each paired comparison ( $\mathrm{AB}$ against $\mathrm{AC} ; \mathrm{AB}$ against $\mathrm{BC}$; AC against $\mathrm{BC}$ ). We compared the experimentally obtained unique fraction with the one of a purely additive scenario created by summing the ensembles recruited by each pathway independently. Strikingly, the uniqueness was almost invariably larger $(t$ test, $p=0.02$ ) between ensembles recruited experimentally as compared to those created digitally (Fig. $4 B)(n=9$ pathway combinations for 3 slices). The ensemble of additional cells re- 
cruited by inputs $\mathrm{AB}$ was markedly different from that recruited by inputs $\mathrm{AC}$ or BC. Indeed, the majority $(85 \pm 3 \%)$ of additional neurons recruited by each combination of inputs were specific for that combination (Fig. $4 B$ ), despite the fact that half of the inputs were common to each combination. Thus, the cooperative recruitment of cortical cell ensembles can enhance differences in the representation of input combinations.

\section{Discussion}

In this study, we examined the recruitment of $\mathrm{L} 2 / 3$ cortical neurons by $\mathrm{M} / \mathrm{T}$ cell inputs. We found in bulb-cortex slices that coactivation of distinct subsets of glomeruli produced responses in more cortical neurons than those recruited by the individual glomerular pathways. This cooperative recruitment of cortical neurons cannot be due to bulbar circuitry alone, because similar results were observed using direct activation of $\mathrm{M} / \mathrm{T}$ cell axons in isolated cortical slices. Furthermore, selective pharmacological blockade of intracortical associational circuitry did not eliminate cooperative recruitment. Together, these results demonstrate that individual cortical neurons are excited by $\mathrm{M} / \mathrm{T}$ cell inputs arising from distinct glomeruli. Our results also indicate that cooperative recruitment only occurs when coactivation of $\mathrm{M} / \mathrm{T}$ cell inputs occur within a narrow time window. Finally, we show that the composition of cooperatively recruited cell ensembles is highly specific for that particular combination of afferent inputs.

What underlies our observations of the recruitment of new pyramidal cells by combinations of stimuli? The simplest explanation for this type of recruitment reflects the divergent nature of connections between $\mathrm{M} / \mathrm{T}$ cell axons and cortical pyramidal cells and the convergence of multiple $\mathrm{M} / \mathrm{T}$ cell inputs onto the same pyramidal cell (Ojima et al., 1984; Franks and Isaacson, 2006; Bathellier et al., 2009). Thus, ensembles of pyramidal cells that receive subthreshold input from different sources will reach spike threshold when inputs are combined. In contrast to other cortical pyramidal cells (London and Häusser, 2005), regenerative events in the apical dendrites of piriform cortex pyramidal cells do not contribute to synaptic integration; rather, spike output is governed by the linear summation of synaptic input at the cell soma (Bathellier et al., 2009). Indeed, the narrow time window for nonlinear recruitment of new cell ensembles is nearly identical to the integration time window of LOT-evoked EPSPs in APC pyramidal cells (Franks and Isaacson, 2006). Associational excitatory inputs formed between pyramidal cells could further enhance supralinear cortical responses.

Our findings are consistent with in vivo studies reporting that odor mixtures can activate larger ensembles of cells than those activated by the individual components (Zou and Buck, 2006; Stettler and Axel, 2009). Furthermore, in vivo recordings of single cells in the anterior olfactory nucleus (another cortical region receiving direct input from $\mathrm{M} / \mathrm{T}$ cells) found that odor mixtures produced stronger depolarizing responses than those predicted from the linear sum of responses to the individual components (Lei et al., 2006). In piriform cortex, odor mixtures have also been found to generate sublinear responses in vivo (Wilson, 2003; Stettler and Axel, 2009). This may result from mixture suppression of OSN responses (Duchamp-Viret et al., 2003) or M/T cell activity (Giraudet et al., 2002; Davison and Katz, 2007) or if nonlinear properties of the cortex are influenced by statedependent, neuromodulatory pathways (Murakami et al., 2005).

Our results establish that the integration of input from different glomeruli by single cortical neurons is a fundamental feature that can shape the representation of sensory information in piriform cortex.

\section{References}

Balu R, Pressler RT, Strowbridge BW (2007) Multiple modes of synaptic excitation of olfactory bulb granule cells. J Neurosci 27:5621-5632.

Bathellier B, Margrie TW, Larkum ME (2009) Properties of piriform cortex pyramidal cell dendrites: implications for olfactory circuit design. J Neurosci 29:12641-12652.

Cang J, Isaacson JS (2003) In vivo whole-cell recording of odor-evoked synaptic transmission in the rat olfactory bulb. J Neurosci 23:4108-4116.

Davison IG, Katz LC (2007) Sparse and selective odor coding by mitral/ tufted neurons in the main olfactory bulb. J Neurosci 27:2091-2101.

Duchamp-Viret P, Duchamp A, Chaput MA (2003) Single olfactory sensory neurons simultaneously integrate the components of an odour mixture. Eur J Neurosci 18:2690-2696.

Franks KM, Isaacson JS (2005) Synapse-specific downregulation of NMDA receptors by early experience: a critical period for plasticity of sensory input to olfactory cortex. Neuron 47:101-114.

Franks KM, Isaacson JS (2006) Strong single-fiber sensory inputs to olfactory cortex: implications for olfactory coding. Neuron 49:357-363.

Giraudet P, Berthommier F, Chaput M (2002) Mitral cell temporal response patterns evoked by odor mixtures in the rat olfactory bulb. J Neurophysiol $88: 829-838$.

Ikegaya Y, Le Bon-Jego M, Yuste R (2005) Large-scale imaging of cortical network activity with calcium indicators. Neurosci Res 52:132-138.

Lei H, Mooney R, Katz LC (2006) Synaptic integration of olfactory information in mouse anterior olfactory nucleus. J Neurosci 26:12023-12032.

London M, Häusser M (2005) Dendritic computation. Annu Rev Neurosci 28:503-532.

Mombaerts P, Wang F, Dulac C, Chao SK, Nemes A, Mendelsohn M, Edmondson J, Axel R (1996) Visualizing an olfactory sensory map. Cell 87:675-686.

Murakami M, Kashiwadani H, Kirino Y, Mori K (2005) State-dependent sensory gating in olfactory cortex. Neuron 46:285-296.

Neville KR, Haberly LB (2004) Olfactory cortex. In: The synaptic organization of the brain, Ed 5 (Shepherd GM, ed), pp 415-454. New York: Oxford UP.

Ojima H, Mori K, Kishi K (1984) The trajectory of mitral cell axons in the rabbit olfactory cortex revealed by intracellular HRP injection. J Comp Neurol 230:77-87.

Poo C, Isaacson JS (2009) Odor representations in olfactory cortex: "sparse" coding, global inhibition, and oscillations. Neuron 62:850-861.

Rennaker RL, Chen CF, Ruyle AM, Sloan AM, Wilson DA (2007) Spatial and temporal distribution of odorant-evoked activity in the piriform cortex. J Neurosci 27:1534-1542.

Royet JP, Souchier C, Jourdan F, Ploye H (1988) Morphometric study of the glomerular population in the mouse olfactory bulb: numerical density and size distribution along the rostrocaudal axis. J Comp Neurol 270:559-568.

Stettler DD, Axel R (2009) Representations of odor in the piriform cortex. Neuron 63:854-864.

Wilson DA (2003) Rapid, experience-induced enhancement in odorant discrimination by anterior piriform cortex neurons. J Neurophysiol 90:65-72.

Wilson RI (2008) Neural and behavioral mechanisms of olfactory perception. Curr Opin Neurobiol 18:408-412.

Wilson RI, Mainen ZF (2006) Early events in olfactory processing. Annu Rev Neurosci 29:163-201.

Zou Z, Buck LB (2006) Combinatorial effects of odorant mixes in olfactory cortex. Science 311:1477-1481. 